\title{
Molecular Motion in Isotropic Turbulence
}

\author{
Jing Fan, Jian-Zheng Jiang, and Fei Fei \\ Laboratory of High Temperature Gas Dynamics, Institute of Mechanics \\ Chinese Academy of Sciences, Beijing 100190, China
}

\begin{abstract}
Molecular motion in turbulence is a major fundamental and engineering problem, and the classical particle approaches are difficult to solve it because of a wide separation of temporal and spatial scales between thermal motion and turbulent fluctuation. A hybrid method named as tracer molecules in continuum (TMIC) has been proposed to address the issues. Using the TMIC method, molecular motion in isotropic turbulence was investigated, and the Reynolds number based on the Taylor micro-scale was about 68. The TMIC calculation shows that tracer molecules are dominated by the thermal motion when time is comparable to the mean collision time, and by the turbulent fluctuation when time is comparable to the integral time scale of turbulence. Between the two time scales, a mixed mode prevails for which both thermal motion and turbulent fluctuation are important. The corresponding turbulent diffusion and viscosity coefficients obtained by TMIC are about $1.2 \times 10^{-3} \mathrm{~m}^{2} \mathrm{sec}^{-1}$ and $7.5 \times 10^{-3} \mathrm{Nm}^{-2} \mathrm{sec}$, respectively, two orders larger than those due to thermal motion
\end{abstract}

Keywords: isotropic turbulence, tracer molecule, TMIC, DNS, DSMC

PACS: 51.10.+y, 47.27.Gs

\section{INTRODUCTION}

Turbulent flows prevail in industrial processes and natural environment that greatly enhances the mass, momentum and energy transport of molecules. Richard Feynman describes turbulence as "the most important unsolved problem of classical physics." Compared to enormous studies on turbulence from the continuum viewpoint, the counterpart at molecular level was much less. The latter is straightforward in principle using particle approaches such as the molecular dynamics (MD) method or the direct simulation Monte Carlo (DSMC) method, but it is not so easy in practice. A big difficulty arises from a wide separation of temporal and spatial scales between molecules and turbulences. It is extremely time-consuming for the particle approaches to resolve turbulent flows directly with the mean free path $\left(\lambda_{m}\right)$ and mean collision time $\left(\tau_{c}\right)$. Even for a simplest case, i.e. homogeneous isotropic turbulence at relatively low Reynolds numbers, the MD or DSMC simulation is beyond the capabilities of current supercomputers.

A hybrid method named as tracer molecules in continuum (TMIC) is proposed to solve the kind of issues. For molecular motion in isotropic turbulence interested here, the direct numerical simulation (DNS) method is firstly employed to solve the turbulent flow field. Then a number of tracer molecules are put in the computational domain of DNS and tracked. When a tracer molecule moves into a cell of DNS, the number density of its surrounding molecules can be obtained directly from the DNS solution, and their velocities are assigned from a local equilibrium distribution whose mean velocity is equal to the turbulent fluctuating velocity in the cell obtained by DNS. For gaseous medium, the tracer molecule can collide with its surrounding molecules following a procedure similar to DSMC [1]. The features of molecular motion in the turbulence are obtained through statistical analysis of the trajectories of the tracer molecules.

In the present paper, the TMIC method is firstly tested in tracking tracer molecules in a three-dimensional box with periodic boundary conditions, and the transport coefficients obtained from the trajectories of the tracer molecules based on the Einstein relation and the Green-Kubo formulas are compared to the theoretical values. Next, TMIC is applied to isotropic turbulence, and the mean square displacement and velocity variation of tracer molecules are compared with the classical theory of turbulence. Further, the diffusion and viscosity coefficients of the isotropic turbulence are determined from the trajectories of the tracer molecules. 


\section{TEST OF TMIC}

Consider argon gas in the standard conditions (273K \& 1atm) and at rest. The computational domain is a periodic box with the side length of $10 \lambda_{m}$, and it is uniformly divided into $20 \times 20 \times 20$ cells. The hard-sphere model is employed to describe the interaction between tracer molecules and their surrounding molecules. Initially, forty tracer molecules are randomly put in each cell. For each time step of $0.3 \tau_{c}$, a tracer molecule moves and collides with its surrounding molecules assigned from a Maxwellian distribution in the standard state. To test TMIC, the transport coefficients are calculated through statistical averaging the trajectories of all the tracer molecules as follows.

According to the Einstein relation [2], the diffusion coefficient is proportional to the mean square displacement of tracer molecules when the time interval $t>\tau_{c}$, i.e.

$$
D=\frac{\left\langle\Delta r^{2}\right\rangle}{6 t}=\frac{1}{6 N t} \sum_{j=1}^{N}\left\{\left[x_{1, j}(t)-x_{1, j}(0)\right]^{2}+\left[x_{2, j}(t)-x_{2, j}(0)\right]^{2}+\left[x_{3, j}(t)-x_{3, j}(0)\right]^{2}\right\} .
$$

According to the Green-Kubo formulas [2], the viscosity and thermal conductivity coefficients have the following expressions

$$
\begin{array}{r}
\mu=\frac{1}{V k T} \int_{0}^{\infty}\left\langle J_{i j}(0) J_{i j}(t)\right\rangle d t, \\
\kappa=\frac{1}{V k T^{2}} \int_{0}^{\infty}\left\langle H_{i}(0) H_{i}(t)\right\rangle d t,
\end{array}
$$

where

$$
J_{i j}(t)=\sum_{k=1}^{N} m \dot{x}_{i, k} \dot{x}_{j, k}, \quad H_{i}(t)=\sum_{k=1}^{N}\left(m u_{i, k}^{2} / 2-5 k T / 2\right) \dot{x}_{i, k}, \quad u_{i}=\dot{x}_{i}=d x_{i} / d t,
$$

$\mathrm{m}$ is the molecular mass, $\mathrm{k}$ is the Boltzmann constant, $\mathrm{V}$ and $\mathrm{T}$ are the volume and temperature of the box, respectively.

In the TMIC method, the correlation of a tracer molecule before and after a collision is taken into account, but the correlation between the tracer molecule and its collision partner is neglected. It can be proven that certain correction factors are necessary for TMIC calculating the viscosity and thermal conductivity based on Eqs. (3) and (4), and they are equal to 4/3 and 59/32, respectively, under the hard-sphere model.

As shown in Fig.1, the transport coefficients obtained by TMIC are consistent with the free molecular prediction when $t<<\tau_{c}$, and agree well with the Chapman-Enskog theory [3] when $t>>\tau_{c}$.
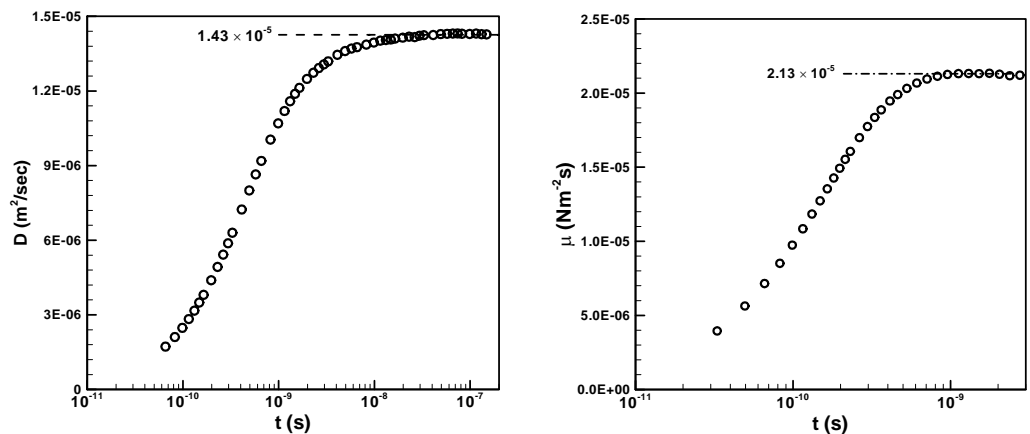

\begin{tabular}{|c|c|c|c|}
\hline \multirow{2}{*}{ Transport coefficient } & \multicolumn{2}{|c|}{ TMIC } & \multirow{2}{*}{ Chapman-Enskog Theory [3] } \\
\hline & Einstein & Green-Kubo & \\
\hline$D\left(\mathrm{~m}^{2} \mathrm{sec}\right)$ & $1.43 \times 10^{-5}$ & - & $1.43 \times 10^{-5}$ \\
\hline$\mu\left(\mathrm{Nm}^{-2} \mathrm{sec}\right)$ & - & $2.13 \times 10^{-5}$ & $2.12 \times 10^{-5}$ \\
\hline$\kappa\left(\mathrm{WK}^{-1} \sec ^{-1}\right)$ & - & $1.71 \times 10^{-2}$ & $1.62 \times 10^{-2}$ \\
\hline
\end{tabular}

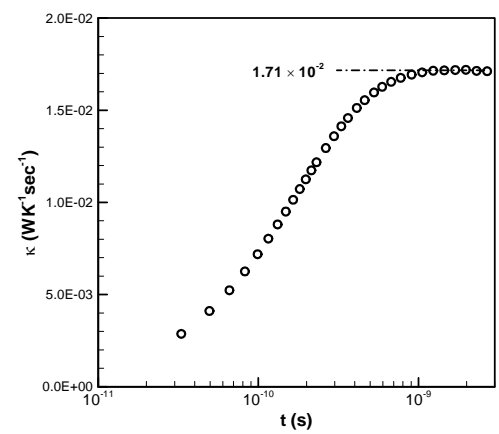

FIGURE 1. Transport coefficients calculated by TMIC. Left: diffusion; center: viscosity; right: thermal conductivity.

TABLE 1. Comparison of transport coefficients of argon gas in the standard conditions 


\section{MOLECULAR MOTION IN ISOTROPIC TURBULENCE}

Consider an isotropic turbulence of argon gas at the standard conditions. The computational domain is a periodic box whose side length is $2 \pi \ell$, and $\ell$ is the integral length scale of turbulence. The Reynolds number based on $\ell\left(\operatorname{Re}_{\ell}=v_{r m s} \ell / v\right)$ is 305, where $v_{r m s}$ is the root mean square (rms) velocity of the turbulent fluctuations. The turbulent Mach number $\left(M a_{t}=v_{r m s} / a\right)$ takes a value of 0.17 , and therefore the incompressible Navier-Stokes equations are approximately valid that are numerically solved by a pseudospectral method for forced turbulence widely used in literatures $[4,5]$. The computational domain is uniformly divided into $128^{3}$ cells for DNS, and the time step is set to be $2 \times 10^{-3} T_{\ell}$, with $T_{\ell}=\ell / v_{r m s}$.

Four different scales are summarized in Table 2. If we introduce the Knudsen number based on the Kolmogorov scale, it is about 0.06 . The Reynolds number based on the Taylor microscale $\left(\operatorname{Re}_{\lambda}=v_{r m s} \lambda_{t} / v\right)$ is about 68. The cell size and time step of DNS are $3.9 \times 10^{-6} \mathrm{~m}$ and $2.6 \times 10^{-9} \mathrm{sec}$, about $62 \lambda_{\mathrm{m}}$ and $16 \tau_{c}$, respectively.

TABLE 2. Comparison of four different scales in isotropic turbulence. The integral scales are determined as follows: $v_{\text {rms }}$ from the values of $M a_{t}$ and the sound speed of argon gas at the standard condition, and $\ell=\operatorname{Re}_{\ell} \times v / v_{\text {rms }}$. The mean energy dissipation rate $\varepsilon=v_{r m s}^{3} / l=2.1 \times 10^{9} \mathrm{~m}^{2} \mathrm{~s}^{-3}$, and the Taylor microscale $\lambda_{t}=\sqrt{15 v v_{r m s}^{2} / \varepsilon}$. The Kolmogorov scales are derived from $\eta=\left(v^{3} / \varepsilon\right)^{1 / 4}, v_{\eta}=(\varepsilon v)^{1 / 4}$, and $\tau_{\eta}=(v / \varepsilon)^{1 / 2}$. The most probable speed of thermal motion $c_{m}=\sqrt{2 k T / m}, \lambda_{m}=1 /\left(\sqrt{2} n \sigma_{T}\right)$ and $\tau_{c} \approx 0.9 \lambda_{m} / \nu_{m}$ under the hard-sphere model with the collision cross section $\sigma_{T}$.

\begin{tabular}{lcccc}
\hline Scale & Integral & Taylor & Kolmogorov & Molecule \\
\hline Length $(\mathrm{m})$ & $6.9 \times 10^{-5}$ & $1.5 \times 10^{-5}$ & $9.5 \times 10^{-7}$ & $6.3 \times 10^{-8}$ \\
Velocity $(\mathrm{m} / \mathrm{s})$ & 52.5 & - & 12.6 & 337 \\
Time $(\mathrm{sec})$ & $1.3 \times 10^{-6}$ & - & $7.5 \times 10^{-8}$ & $1.6 \times 10^{-10}$ \\
\hline
\end{tabular}

\subsection{Mean Square Displacement}

The displacement of a tracer molecule in the $\mathrm{x}$ direction can be written as

$$
\Delta x(t)=\int_{0}^{t}\left[c_{x}(t)+v_{x}(t)\right] d t .
$$

If the velocities of the thermal motion and turbulent fluctuation, $c_{x}(t)$ and $v_{x}(t)$, are irrelevant, then

$$
\left\langle\Delta x^{2}\right\rangle=\int_{0}^{t} \int_{0}^{t}\left\langle\left[c_{x}(\tau)+v_{x}(\tau)\right]\left[c_{x}\left(\tau^{\prime}\right)+v_{x}\left(\tau^{\prime}\right)\right]\right\rangle d \tau d \tau^{\prime}=\left\langle\Delta x_{m}^{2}\right\rangle+\left\langle\Delta x_{t}^{2}\right\rangle .
$$

The first term on RHS has the following exact solutions in free molecular and continuum limits [2]

$$
\left\langle\Delta x_{m}^{2}\right\rangle=\int_{0}^{t} \int_{0}^{t}\left\langle c_{x}(\tau) c_{x}\left(\tau^{\prime}\right)\right\rangle d \tau d \tau^{\prime}=\left\{\begin{array}{ll}
c_{m}{ }^{2} t^{2} & t<<\tau_{c} \\
2 D t & t>>\tau_{c}
\end{array},\right.
$$

The second term on RHS arises from turbulent fluctuation has the expressions similar to (7), but the characteristic time becomes $T_{\ell}$ instead of $\tau_{c}[6]$

$$
\left\langle\Delta x_{t}^{2}(t)\right\rangle=\int_{0}^{t} \int_{0}^{t}\left\langle v_{x}(\tau) v_{x}\left(\tau^{\prime}\right)\right\rangle d \tau d \tau^{\prime}=\left\{\begin{array}{ll}
v_{r m s}^{2} t^{2} / 3 & t<<T_{\ell} \\
2 v_{r m s}^{2} T_{\ell} t / 3 & t>>T_{\ell}
\end{array} .\right.
$$

Substitution of (7) and (8) into (6), together with a kinetic relation $D=3 c_{m}^{2} \tau_{c} / 4$, yields

$$
\frac{\left\langle\Delta x^{2}\right\rangle}{2 t}=\left\{\begin{array}{lc}
\left(3 c_{m}{ }^{2}+2 v_{r m s}^{2}\right) t / 12 & t<<\tau_{c} \\
\left(9 c_{m}^{2} \tau_{c}+2 v_{r m s}^{2} t\right) / 12 & \tau_{c}<<t<<T_{\ell} \\
\left(9 c_{m}^{2} \tau_{c}+4 v_{r m s}^{2} T_{\ell}\right) / 12 & t>>T_{\ell}
\end{array}\right.
$$


Figure 2 shows the mean square displacement of all tracer molecules in isotropic turbulence. It agrees well with Eq. (9) at the different time scales.

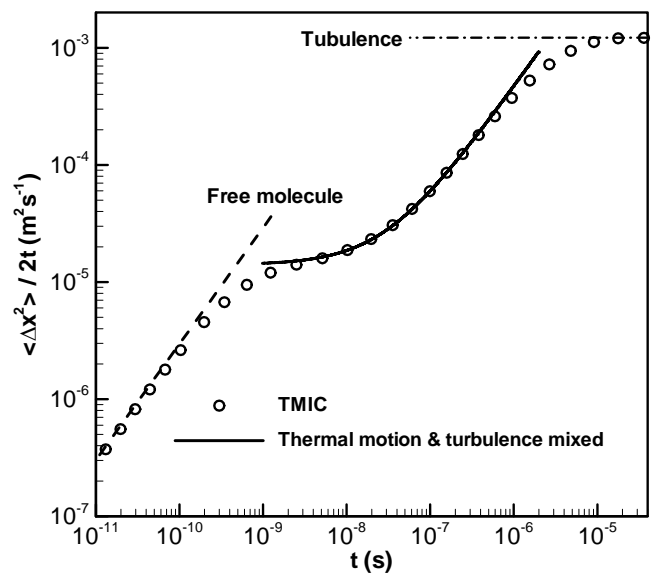

FIGURE 2. Variation of the mean square displacement of tracer molecules in isotropic turbulence $\left(\operatorname{Re}_{\lambda}=68\right)$ with time.

\subsection{Mean Square Velocity Variation}

The x component of the mean square thermal velocity variation of a tracer molecule can be written as

$$
\left\langle\left[c_{x}\left(t+t_{0}\right)-c_{x}\left(t_{0}\right)\right]^{2}\right\rangle=\left\langle c_{x}^{2}\left(t+t_{0}\right)\right\rangle+\left\langle c_{x}^{2}\left(t_{0}\right)\right\rangle-2\left\langle c_{x}\left(t+t_{0}\right) c_{x}\left(t_{0}\right)\right\rangle .
$$

In an equilibrium state, we have

$$
\left\langle c_{x}\left(t+t_{0}\right) c_{x}\left(t_{0}\right)\right\rangle=\left\langle c^{2}{ }_{x}\left(t_{0}\right)\right\rangle e^{-\zeta t}, \quad\left\langle c_{x}^{2}\left(t_{0}\right)\right\rangle=\left\langle c_{x}^{2}\left(t+t_{0}\right)\right\rangle=0.5 c_{m}^{2}
$$

where $\zeta=k T /(m D)=\alpha / \tau_{c}$, and $\alpha=2 / 3$.

Substitution of (11) into (10) yields

$$
\left\langle\left[c_{x}\left(t+t_{0}\right)-c_{x}\left(t_{0}\right)\right]^{2}\right\rangle=c_{m}^{2}\left(1-e^{-\alpha t / \tau_{c}}\right) .
$$

According to the Kolmogorov's small-scale similarity theory [6], the x component of the second-order autocorrelation function of turbulent fluctuation velocity has an expression as follows

$$
\left\langle\left[v_{i}\left(t+t_{0}\right)-v_{i}\left(t_{0}\right)\right]^{2}\right\rangle=\left\{\begin{array}{ll}
A_{0} \varepsilon^{1.5} v^{-0.5} t^{2} & t<<t_{\eta} \\
C_{0} \varepsilon t & t_{\eta}<<t<<T_{\ell}
\end{array}= \begin{cases}A_{0} v_{\eta}^{2}\left(t / \tau_{\eta}\right)^{2} & t<<\tau_{\eta} \\
C_{0} v_{\eta}^{2} \times t / \tau_{\eta} & t_{\eta} \ll t<<T_{l}\end{cases}\right.
$$

where $A_{0}$ and $C_{0}$ are constants.

The velocity of a tracer molecule due to thermal motion and turbulent fluctuation velocities are assumed irrelevant, then we have

$$
\left\langle\Delta u^{2}\right\rangle=\left\langle\left[c_{i}\left(t+t_{0}\right)-c_{i}\left(t_{0}\right)\right]^{2}\right\rangle+\left\langle\left[v_{i}\left(t+t_{0}\right)-v_{i}\left(t_{0}\right)\right]^{2}\right\rangle=\left\{\begin{array}{ll}
c_{m}^{2}\left(1-e^{-\alpha t / \tau_{c}}\right)+A_{0} v_{\eta}^{2}\left(t / \tau_{\eta}\right)^{2} & t<<\tau_{\eta} \\
c_{m}^{2}\left(1-e^{-\alpha t / \tau_{c}}\right)+C_{0} v_{\eta}^{2} \times t / \tau_{\eta} & \tau_{\eta}<<t<<T_{\ell}
\end{array} .\right.
$$

Figure 3 shows the mean square velocity variation of all tracer molecules in isotropic turbulence. It is in good agreement with Eq. (14) when $A_{0}=5$, and $C_{0}=2$. See Ref. [7] for a detailed discussion on the values of $A_{0}$ and $C_{0}$.

\section{TURBULENT DIFFUSION AND VISCOSITY COEFFICIENTS}

The Einstein relation (1) is not only applied to thermal motion, but also to turbulent fluctuation. Based on the relation, the diffusion coefficient of isotropic turbulence can be obtained from the mean square displacement of tracer molecules in the long time limit. From Fig.1, it is about $1.2 \times 10^{-3} \mathrm{~m}^{2} \mathrm{sec}^{-1}$ when $\operatorname{Re}_{\lambda}=68$. This value is two orders larger than the diffusion coefficient due to thermal motion $\left(1.43 \times 10^{-5} \mathrm{~m}^{2} \mathrm{sec}^{-1}\right)$.

The Green-Kubo formula for viscosity is extended to apply for isotropic turbulence. Under the assumption that the thermal motion and turbulent fluctuation velocities of tracer molecules to be irrelevant, Eq. (3) is simplified as 


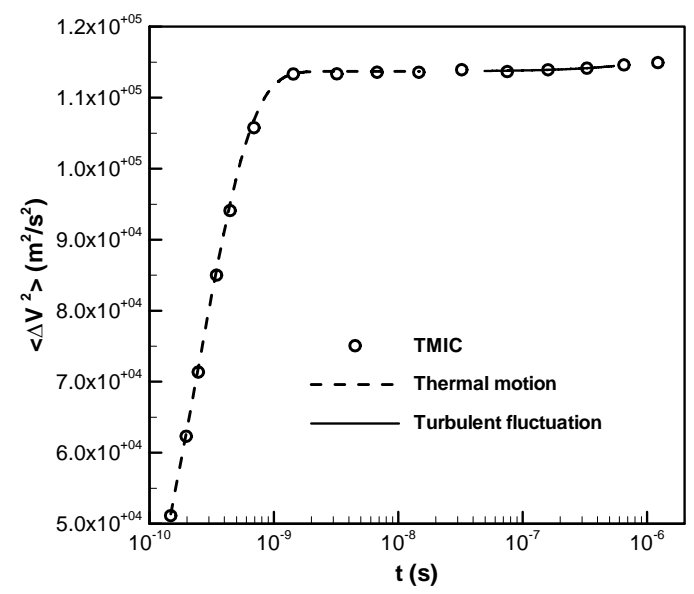

FIGURE 3. Variation of the mean square velocity of tracer molecules in isotropic turbulence $\left(\operatorname{Re}_{\lambda}=68\right)$ with time.

where

$$
\mu=\mu_{m}+\mu_{t},
$$

$$
\begin{array}{cc}
\mu_{m}=\frac{1}{V k T} \int_{0}^{\infty}\left\langle J_{i j}^{m}(0) J_{i j}^{m}(t)\right\rangle d t, \quad J_{i j}^{m}(t)=\sum_{k=1}^{N} m c_{i, k} c_{j, k}, \\
\mu_{t}=\frac{1}{V k T} \int_{0}^{\infty}\left\langle J_{i j}^{t}(0) J_{i j}^{t}(t)\right\rangle d t, & J_{i j}^{t}(t)=\sum_{k=1}^{N} m v_{i, k} v_{j, k} .
\end{array}
$$

In the present DNS calculation of forced turbulence, the values of kinetic energy are fixed for the wave number below 4. Such a common treatment leads to an anisotropic effect on the large scale. Eq. (17) is modified as follows to reduce the effect.

$$
\mu_{t}=\frac{1}{V k T} \int_{0}^{\infty}\left\langle C_{i j}^{t}(0) C_{i j}^{t}(t)\right\rangle d t, \quad C_{i j}(t)=J_{i j}^{t}(t)-\overline{J_{i j}^{t}(t)},
$$

with

$$
\overline{J_{i j}^{t}(t)}=\frac{1}{M} \sum_{k=1}^{M} J_{i j, k}^{t}(t)
$$

Figure 4 shows the evolution of viscosity coefficients as well as $C_{i j}(t)$ in isotropic turbulence. The mean value of the viscosity coefficients due to turbulent fluctuations is about $7.5 \times 10^{-3} \mathrm{Nm}^{-2} \mathrm{sec}$ when $\operatorname{Re}_{\lambda}=68$. It is two orders larger than the thermal viscosity coefficient of argon gas $\left(2.1 \times 10^{-5} \mathrm{Nm}^{-2} \mathrm{sec}\right)$. Because of the anisotropy of the forced turbulent on the large scale, the turbulent viscosity coefficients are somewhat different in the three directions.
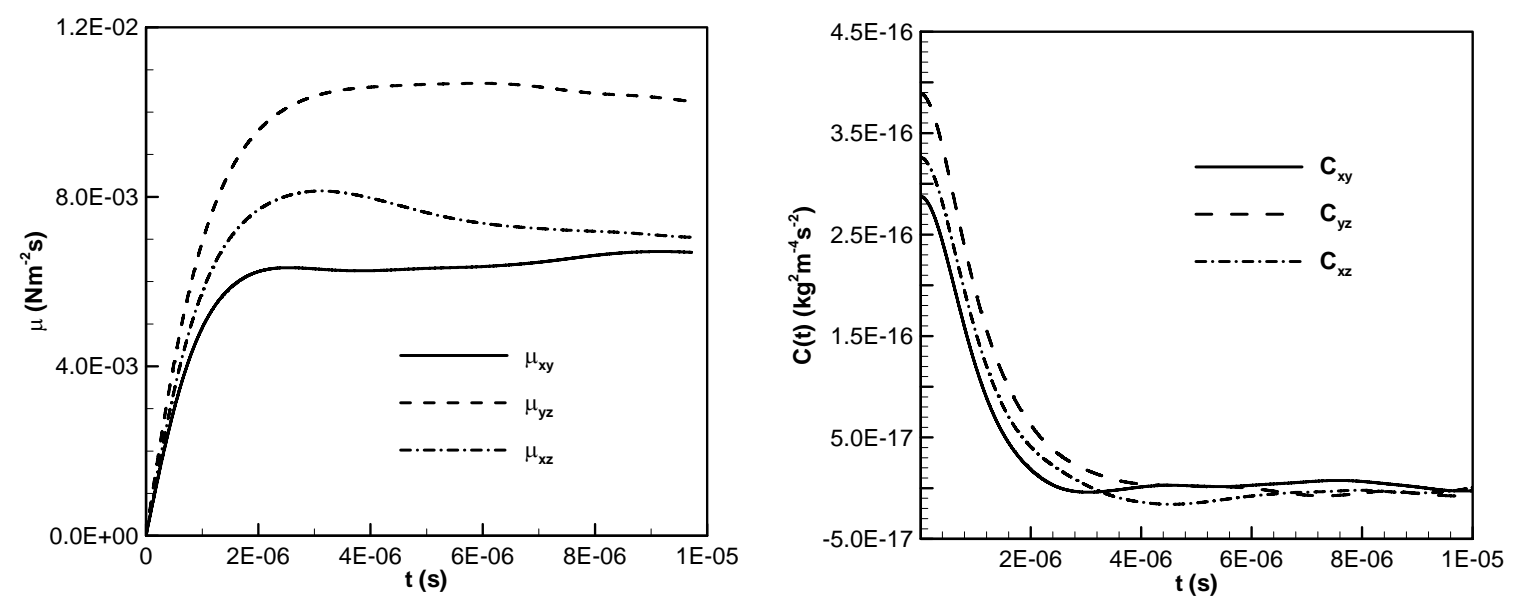

FIGURE 4. Variation of the viscosity coefficients and their correlation functions in isotropic turbulence $\left(\operatorname{Re}_{\lambda}=68\right)$ with time. 


\section{DISCUSSIONS}

The TMIC method provides a tool to observe and understand molecular motion in turbulence. For an isotropic turbulence case studied here, typically shown by Fig. 2 and Eq. (9), tracer molecules are dominated by the thermal motion when time is comparable to the mean collision time, and by the turbulent fluctuation when time is comparable to the integral time scale of turbulence. Between the two time scales, a mixed mode prevails for which both thermal motion and turbulent fluctuation are important.

Compared with the notion of fluid element widely used in fluid mechanics, molecules are a reality, and the interaction between them, at least for gases in a room state, are clearly understood. Figure 2 and Eq. (9) suggest an analogy between turbulent fluctuation and thermal motion: the root mean square velocity, integral length and time, in the viewpoint of diffusion, correspond to the thermal velocity, mean free path and mean collision time, respectively. At least for isotropic turbulence, such an analogy has a true physical meaning.

More complicated turbulent flows are composed by "eddies" of different sizes. A cascade process was suggested by Richardson [8]: "Big whirls have little whirls, which feed on their velocity; little whirls have smaller whirls, an so on to the viscosity.” In this way, the energy is passed down from the large scales of the motion to smaller scales until reaching a sufficiently small length scale such that the viscosity of the fluid can effectively dissipate the kinetic energy into internal energy. To a certain extent, a universal picture for molecular motion in the cascade process is equivalent to the closure problem of turbulence in continuum mechanics. This is a very important issue that needs more efforts in future.

\section{ACKNOWLEDGMENTS}

This work was supported by the National Natural Science Foundation of China under Grant No. 10921062. The authors thank Professor X. L. Li who provided a DNS code and valuable discussion on isotropic turbulence.

\section{REFERENCES}

1. G. A. Bird, Molecular Gas Dynamics and the Direct Simulation of Gas Flows, Oxford: Clarendon Press, 1994

2. D.A.Mcquarrie, Statistical Mechanics, New York: Harper \& Row, 1973, pp. 512-524.

3. S. Chapman and T. G. Cowling, The Mathematical Theory of Non-uniform Gases, $3^{\text {rd }}$ ed., Cambridge: Cambridge Univ. Press, 1970.

4. S. A. Orszag and G. S. Patterson, Phys. Rev. Lett., 28, 76 (1972)

5. T. Ishihara, T. Gotoh, Y. Kaneka, Ann. Rev. Fluid Mech., 41,106-180 (2009)

6. A. S. Monin, A. M. Yaglom, Statistical Fluid Mechanics, Cambridge: MIT Press, 1975

7. P. K. Yeung, Annu. Rev. Fluid Mech., 34, 115-42 (2002).

8. L.F. Richardson, Whether Prediction by Numerical Process, Cambridge: Cambridge Univ. Press, 1922. 